\title{
Anemia Megaloblástica en la infancia: 3 casos de diferente Etiología
}

\author{
DRS.: DANIEL SANTANDER S., RAFAEL DlCOS S., HERNAN TABOADA L. *
}

Se entiende por Anemia Megaloblástica (A.M.), una entidad morfológica característica producida por un defecto en la síntesis de núcleo-proteínas, determinado por diversas causas. Se manifiesta por la presencia de células progenitoras gigantes en la médula hematopoyética, y macro-ovalocitos, polinucleares hipersegmentados y plaquetas gigantes en la sangre (28).

Más de un $95 \%$ de las A.M. es debido a un déficit de Acido Fólico (AF) y/o vitamina $\mathrm{B} 12(29,33)$. En el pequeño grupo restante se encuentran la aciduria orótica congénita, la anemia por déficit de piridoxina, el síndrome de Lesch-Nyhan, la anemia megaloblástica tiamino-dependiente, etc. (29, 72.

La anemia por déficit de vitamina B 12, secundario a ausencia congénita de Factor Intrínseco de la mucosa gástrica, se manifiesta precozmente en el niño, constituyendo la llamada Anemia Perniciosa Juvenil Congénita. Es una enfermedad extraordinariamente rara, ya que hasta el año 1970 sólo se habían descrito alrededor de 30 casos (67, $56,8,48,32)$. El déficit de A.F., en cambio, es el responsable de la gran mayoría de las A.M. en la edad infantil, ocupando el segundo lugar dentro de las anemias deficitarias, a continuación de la ferropriva (70). Más aún, si se toman en cuenta los niveles séricos de folatos, con o sin anemia concomitante, el déficit de A.F. podría ser considerado como la hipovitaminosis más frecuente $(47,31)$. De hecho, constituye un problema de salud mundial, especialmente importante en países en desarrollo, ya que afecta con gran frecuencia a las capas sociales de bajos ingresos $(33,36,30,23,19,42)$.

\footnotetext{
* Servicio de Pediatría, Hospital Sótero del Río. Departamento de Pediatría, Universidad Católica de Chile.
}

Si bien el aporte dietético insuficiente es la causa más frecuente de déficit de A.F., existen otros mecanismos por los cuales este último puede ocurrir, entre los que hay que destacar: 1) Absorción inadecuada, secundaria a Enfermedad Celíaca u otras alteraciones de la mucosa intestinal $(29,9,33$, $52,45,16,12,1,70,49)$, o a ingestión de drogas anticonvulsionantes $(41,79,13)$. 2) Requerimientos aumentados, como ocurre en el embarazo $(36,33,34)$, en la lactancia materna (31), durante el primer año de vida $(73,70,34)$, especialmente en niños con bajo peso de nacimiento (71), en las anemias hemolíticas crónicas $(11,60,15,17,37)$, infecciones $(51,37,76,43,24,54)$, procesos neoplásicos (28), hipertiroidismo (34) y en la desnutrición en período de recuperación (82). 3 ) Utilización inadecuada (bloqueo metabólico), especialmente por el uso de antifólicos $(60,68,40,29,79,37)$ y por enfermedades hepáticas $(28,81,57,83)$.

En un período de 6 meses se han presentado en nuestro Servicio 3 casos de Anemia Megaloblástica, obedeciendo a diferentes factores etiológicos. Por este motivo, consideramos de interés, presentar las características clínicas, y hematológicas, y la respuesta terapéutica de dichos pacientes.

\section{CASOS CLINICOS}

\section{$\begin{array}{lllll}\text { C A S O } & N^{\circ} & 1\end{array}$}

J.R. Edad: 8 meses. Paciente de sexo masculino, que ingresa a nuestro Servicio por presentar fiebre de una semana de evolución, agregándose compromiso del estado general y respiración quejumbrosa. En el examen físico se encuentra un lactante febril, desnutrido, con un peso de $4.800 \mathrm{~g}$ 
( $36 \%$ de déficit para el percentil correspondiente), sin edemas, con intensa palidez cutáneo-mucosa y gran decaimiento. Resto del examen negativo. Se practica estudio hematológico de urgencia, encontrándose los siguientes valores: Hb, $4.7 \mathrm{~g} \%$, Hto. $13 \%$ y $54 \%$ de reticulocitos. Al frotis se aprecia normocromía, anisopoiquilocitosis, abundantes macro-ovalocitos e hipersegmentación de los neutró. filos, con un promedio de lobulación de 4.05 (normal $3.17+0.25$ ). El mielograma confirma francas alteraciones megaloblásticas. Con el diagnóstico de Anemia Megaloblástica, se inicia tratamiento con Acido Fólico oral $1 \mathrm{mg}$ diario.

En vista que la madre no concurriera a propor. cionar los datos anamnésticos, se efectúa visita domiciliaria, obteniéndose los siguientes antecedentes: madre de 38 años, postrada en cama desde hace 8 meses, por cuadro de naturaleza no precisada, estando el paciente al cuidado de una hermana de 10 años. Viven en casa de adobe con piso de tierra, ubicada en las afueras de San Bernardo, sin agua potable, luz eléctrica ni alcantarillado. Padre inquilino, bebedor habitual. 5 hermanos vivos, 2 fallecidos por causa no precisada.

Entre los antecedentes personales hay que destacar un parto normal de término, con peso de nacimiento de $2.800 \mathrm{~g}$. Alimentado 2 meses con pecho exclusivo, y posteriormente con Leche Nido ( \pm 120 c.c. 4 veces al día) durante 5 meses. Desde 1 mes antes de su ingreso recibía leche de vaca en igual cantidad, hervida en forma prolongada. Nunca se le había dado jugos, sopas, ni otros alimentos sólidos o suplementos vitamínicos.

Dentro del estudio practicado destacan los siguientes exámenes: proteinemia de $6.46 \mathrm{~g} \mathrm{\%}$, test de Coombs directo negativo, screening de Glucosa-6, Fosfato Dehidrogenasa y Piruvato-kinasa en hematíes normales, ferremia de 185 microgramos $\%$, con transferrina de 350 microgramos \% y saturación de 52\%. Una radiografía ósea demostró signos de raquitismo, por lo que se administró vitamina D2 600.0000 U. Desde su ingreso recibió también vitamina $C 100 \mathrm{mg}$ diarios.

A los 3 días de iniciado el tratamiento se apreció intensa respuesta reticulocitaria (279\%), y las cifras de Hb. y Hto. iniciaron un ascenso progresivo hasta la tercera semana, en que se pudo apreciar una detención relativamente mayor para la Hb. que para el Hto., con una concentración de Hb. globular de $30 \%$ y aparición de signos de hi. pocromía en sangre periférica. Una nueva ferremia fue de 26 microgramos \%, con transferrina de 450 miecrogramos, y saturación de sólo 6\%. Ante estos hallazgos, se consideró que se había manifestado una ferropenia, suspendiendo el ácido fólico (administrado durante 20 días) y agregando Sulrato Ferroso $300 \mathrm{mg}$ diarios. Con ello se pudo apreciar una nueva elevación de los valores hematimétricos, los que al momento de su alta eran de 11 $\mathrm{g} \%$ de Hb. y $36 \%$ de Hto. Al frotis sanguíneo se cvidenciaba solamente anisocromía, y el promedio de lobulación de los neutrófilos había descendido a 3.36 .

Por la curva febril que presentaba el paciente a su ingreso, se practicó Punción Vesical, obteniéndose 2000 colonias por c.c. de E. Coli y Proteus, tratándose con Furadantina según antibiograma.

Diagnóstico final 1) Desnutrición de $2^{\circ}$ grado (calórica). 2) Raquitismo. 3) Infección Urinaria. 4) Anemia Megaloblástica por déficit nutricional de ácido fólico.

\section{$\begin{array}{llllllllll}C & A & S & O & N & : & 2\end{array}$}

M.R. Edad: 10 años 8 meses. Paciente de sexo femenino, portadora de una Leucemia Linfoblástica, en control en Hematología desde hace 2 años. El diagnóstico se hizo por el cuadro clínico, estudio hematológiro y citoquímico en septiembre de 1970. Su tratamiento inicial consistió en inducción de la remisión con Prednisona y 6-mercaptopurina (Purinethol R), durante 1 mes. Obtenida ésta, se inició terapia de mantención con 6-mercaptopu. rina, amethopterina (Methotrexato $\mathrm{R}$ ) y ciclofosfamida (Endoxán R) asociadas o en forma clínica. Se realizó además una reinducción con vincristina (Oncovín R) y prednisona al año de tratamiento. Desde la iniciación de su enfermedad hasta le fecha, la paciente no ha presentado evidencias clínicas ni hematológicas de recaída de su enfermedad.

La complicación terapéutica que deseamos presentar se inició al año y tres meses de tratamiento. Con motivo de un cambio en nuestro esquema de tratamiento de la leucemia linfoblástica, en el que se contempla el uso a permanencia de amethopterina bisemanal, asociada a 6-mercaptopurina $\mathrm{y}$ ciclofosfamida agregados en forma clínica cada 3 meses, la paciente empezó a recibir 6-mercaptopurina en dosis de $50 \mathrm{mgr}$. diarios por vía oral $(2.0 \mathrm{mgr} / \mathrm{Kg}$ ) y amethopterina $17.5 \mathrm{mgr} 2$ veces a la semana, oral $(0.75 \mathrm{mgr} / \mathrm{Kg})$. Después de 6 dosis de esta última droga, se constató una anemia moderada, por lo que se debió suspender, manteniéndose la 6-mercaptopurina. Cuarenta días después, en vista de la recuperación de los valores de $\mathrm{Hb}$. y Hto., se reinicia el tratamiento combinado. Treinta días después, vuelve a presentar anemia moderada, por lo que se continúa solamente con 6-mercaptopurina, droga que es necesario suspender posteriormente por leucopenia de 2.000 por $\mathrm{mm}^{3}$, permaneciendo sin tratamiento durante 14 días, al cabo de los cuales se reinicia terapia con amethopterina, que no recibía desde 45 días. Después de 2 semanas de tratamiento ( 4 dosis orales de $0.75 \mathrm{mgr} / \mathrm{Kg}$ ), presenta anemia de $8,8 \mathrm{grs} \%$ de Hb. y $25 \%$ de Hto. Se practica mielograma, que demuestra una marcada hipoplasia eritroide con franca megaloblastosis, sin apreciarse signos de 
recaída leucémica. Se suspende la droga nuevamente, pero a los 7 días la anemia había aumen. tado a $6.6 \mathrm{grs} \%$ de $\mathrm{Hb}$, y $19 \%$ de Hto. Se decide iniciar tratamiento con Acido N.5 formil-tetrahidrofólico (Acido Folínico, Leucovorina R) en dosis de 3 mgrs I.M. cada 12 horas durante 8 días. Con dicho tratamiento se apreció un ascenso de la Hb. y Hto, que a los 14 días era de 8.8 grs\% y $26 \%$ respectivamente. Se inició entonces un ciclo de reinducción con prednisona, llegando la $\mathrm{Hb}$. a 12.65 grs $\%$ y el Hto. a $38 \%$, con 10.700 leucocitos por $\mathrm{mm}^{3}$ a los 15 días. Posteriormente se completó la reinducción con 3 dosis de vincristina.

$\mathrm{La}$ enferma se encuentra actualmente en buenas condiciones generales $\mathrm{y}$ hematológicas, realizando una vida normal.

\section{A S O N $: 3$}

M.Y. Edad: 7 años 5 meses. Sexo: masculino. Paciente portador de una Anemia Hemolítica Crónica no Esferocítica, por déficit congénito de Glucosa 6 Fosfato Dehidrogenasa, en control en $\mathrm{He}$ matología desde los tres meses de edad. Entre sus antecedentes cabe destacar: Enfermedad Hemolítica del R.N., sin incompatibilidad Rh ni por grupo clásico, que requirió recambio sanguíneo. 3 hermanos varones fallecidos por ictericia neonatal. 7 hermanas vivas sanas. Durante el primer año de vida presenta crisis hemolíticas a repetición, que hacen necesario el uso de transfusiones. Por medio del test de estabilidad del glutatión se diagnostica un déficit congénito de G-6PD en los hematíes. A los 2 años se practica determinación cuantitativa de este enzimo, encontrándose actividad enzimática cercana a 0 . El caso fue publicado anteriormente (45). En la evolución del paciente cabe destacar que ocasionalmente, pese a no recibir ningún tipo de drogas oxidantes, presenta crisis hemolíti. cas generalmente en relación con infecciones virales. Además, en los repetidos controles efectuados, se encuentra reticulocitosis que oscila entre 7 y $20 \%$, aun cuando los valores de $\mathrm{Hb}$. y Hto. sean normales. Con las crisis hemolíticas las cifras de reticulocitosis aumentan, llegando en una ocasión a $70 \%$.

El episodio que nos interesa relatar se presentó a los 7 años 5 meses de edad, después de haber permanecido durante un año en buenas condiciones, controlándose en Hematología cada 6 meses. 6 días antes de su ingreso, empieza a presentar fiebre, coriza, palidez, decaimiento intenso, anorexia y orinas oscuras. Por aumento rápido de esta sintomatología, agregándose somnolencia marcada, es llevado a los dos días a Posta infantil en estado agónico, recibiendo una transfusión de 500 c.c. de sangre. En dicha posta se le practica al día siguiente una $\mathrm{Hb}$., que es de 5.2 grs\%. Se coloca nueva transfusión de 500 c.c. y se solicita traslado a nuestro Servicio. Se recibe paciente consciente, muy decaído, con intensa palidez de piel y mucosas, afebril, en buen estado nutritivo (peso $20 \mathrm{kgs}$., talla $117 \mathrm{cms}$.). En el resto del examen físico se aprecia solamente hepatomegalia de 3 cms., indolora, y bazo (-). El hemograma practicado al ingreso indica: Hb. 7.1 grs\%, Tto. 20\%, reticulocitos $0.3 \%$, leucocitos 2.500 por $\mathrm{mm}^{3}$, plaquetas disminuidas al frotis, fórmula leucocitaria normal, anisocitosis, esquistocitos escasos y normocromía. Se practica mielograma, que informa una hipoplasia eritroide intensa, con relación granulocítico-eritroide de 1:1 (normal 3:1), y marcadas alteraciones de la maduración, consistentes en asincronismo núcleo-citoplasmático, aumento de la proporción de proeritroblastos y eritroblastos basófilos, vacuolización de la serie eritroide y granulocítica, regular cantidad de megaloblastos, y megacariocitos presentes, con disminución de entrega de plaquetas.

Con el diagnóstico de Anemia Megaloblástica complicando una Anemia Hemolítica crónica no Esferocítica, se inicia tratamiento con Acido Fólico $1 \mathrm{mgr}$ diario por vía oral. A los 3 días de tratamiento, la $\mathrm{Hb}$. es de 8.2 grs $\%$ y el Hto. de $23 \%$ y los reticulocitos $3.4 \%$. A los 6 días, hay reticulocitosis de $29.5 \%$, con $\mathrm{Hb}$. de 9.9 grs\% y Hto. de $28 \%$. La evolución es muy satisfactoria, con mejoría subjetiva importante. El último control, a los 14 días de tratamiento con A.F., indica Hb. de 11 grs $\%$, Hto. $37 \%$ y reticulocitos $9.9 \%$.

La conducta futura con este paciente contem. pla el uso de A.F. a permanencia, en dosis de 0.5 mgr diarios, como prevención de otra crisis megaloblástica.

Comentario. Como ya se dijo en la introducción, los casos clínicos relatados constituyen 3 tipos diferentes de A.M. por déficit de A.F.: el primero de ellos corresponde a una falla nutricional, el segundo a un bloqueo metabólico por antagonistas del A.F., y el tercero a un exceso de consumo por un proceso hemolítico crónico.

En la figura No 1 se esquematiza el metabolismo del A.F., haciendo hincapié en los sitios donde pueden existir interferencias.

Nos referiremos en primer término al déficit de aporte en la dieta. La principal fuente dietética de A.F. está formada por los siguientes alimentos: hígado, riñón, legumbres, verduras frescas, frutas cítricas frescas y sus jugos $(72,62,29,70,71)$, y la leche materna (55).

El requirimiento mínimo diario en adultos es de +50 microgramos $(27,38)$; en niños es de 20-50 microgramos $(73,31,34)$, siendo por lo tanto, alrededor de 6 veces mayor que en adultos, en relación al peso.

Se sabe que el A.F. existe en los alimen- 


\section{CELLLA NTESTINAL}

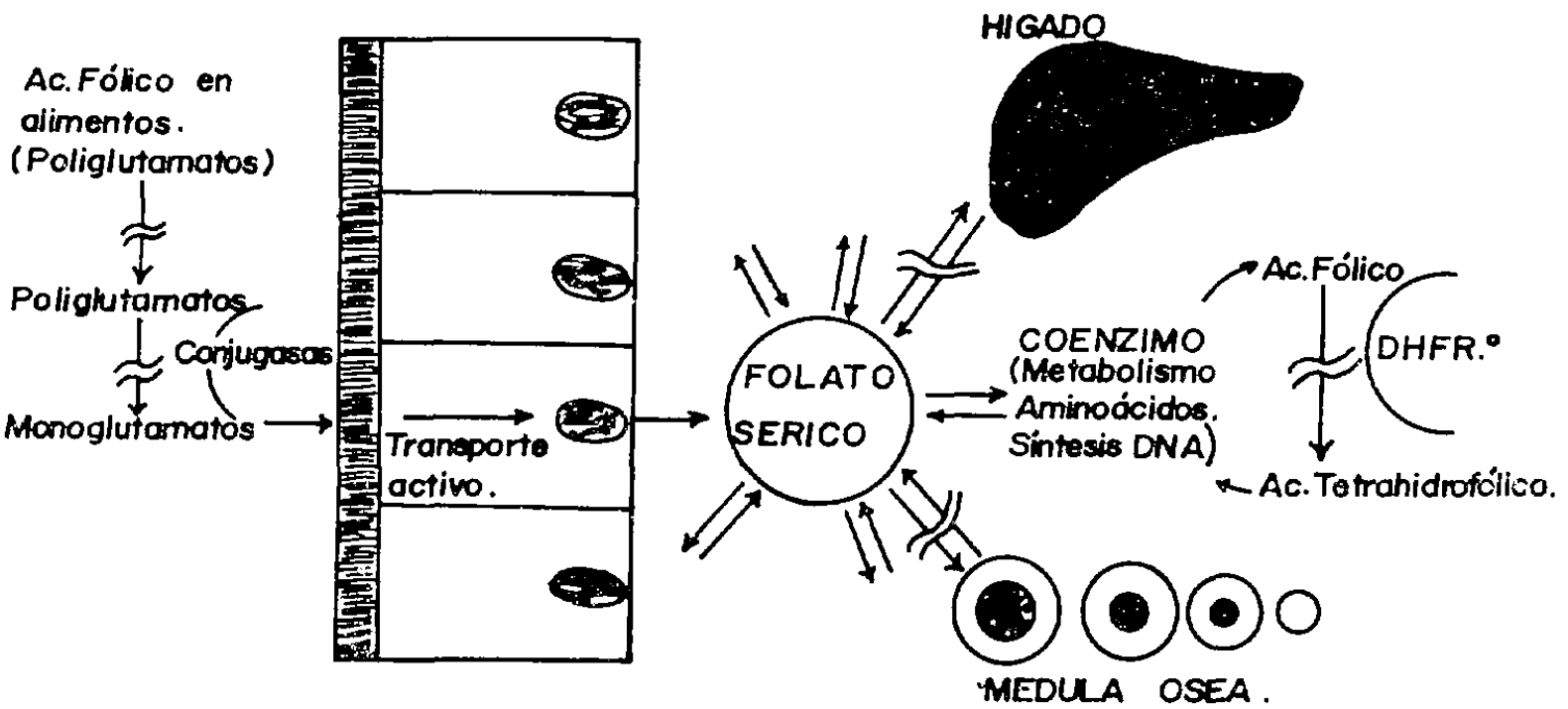

$\begin{aligned} \text { DHFRO } & =\text { DIHIDROFOLICO REDUCTASA. } \\ \pi_{i} & =\text { SITIOS CONOCDOS DE INTERFERENCIA EN EL METABOLISMO. }\end{aligned}$

tos en forma de poliglutamatos $(69,6,62,2)$, los que presentan gran labilidad al calor, por lo cual son fácilmente inactivados por la cocción prolongada y por los procedimientos tecnológicos de conservación $(28,29,31$, $34,33,6,36,70,32)$. Por esta misma razón. la leche de vaca, que tiene un contenido de A.F., similar al de leche humana, pierde más de un $80 \%$ de éste antes de su utilización $(55,6,22)$.

En nuestro primer paciente se encuentra claramente un déficit en el aporte dietético de A.F., ya que sólo fue alimentado al pecho durante 2 meses, recibiendo posteriormente leche en polvo y luego leche de vaca sometida a la cocción, ambas en cantidades insuficientes. Nunca recibió jugos, frutas, ni otros alimentos ricos en A.F.

Continuando con el análisis del Gráfíco, podemos ver que un segundo punto de interferencia en el metabolismo del A.F. existe en el proceso de ingestión y absorción de esta vitamina, que se realiza en el tercio superior del intestino delgado $(28,32,9,29)$. A ese nivel, los poliglutamatos deben ser transformados en monoglutamatos por enzimas denominadas conjugasas, que son producidas por la célula de la mucosa intesti- nal $(6,69,62,5)$. Por este motivo, las alteraciones del intestino delgado, en especial la Enfermedad Celíaca y otras causas de Síndrome de Malabsorción, pueden conducir a A.M. por déficit de A.F. $(9,1,12,80,63,52$, $45,16,29)$. La A.M. secundaria al uso de algunas drogas anticonvulsivantes, especialmente la difenilhidantoína, se debería también a una interferencia en la absorción, por inhibición de las conjugasas $(13,79)$.

Como curiosidad habría que mencionar la reciente descripción de casos de malabsorción congénita de A.F. (46). Además, se ha descrito la posibilidad de aparición de un síndrome de malabsorción con alteraciones histológicas de la mucosa intestinal, secundario a carencia de A.F. $(14,20,64)$, aunque estudios experimentales en ratas no apoyan este hallazgo (44).

Una vez que el A.F. se ha incorporado al organismo, debe participar como coenzimo en una gran cantidad de procesos bioquímicos, mediante la recepción y entrega de unidades de 1 átomo de carbono, siendo el más importante de éstos su intervención en la síntesis de Acidos Nucleicos (50, 68, 7, 28, $60,37,30,33,74)$. Para poder realizar estas funciones, el A.F. debe ser reducido a ácido 
dihidrofólico y tetrahidrofólico, siendo este último la forma activa. En esta reacción actúa una enzima, la dihidrofólicoreductasa, que se encuentra en todos los tejidos, especialmente en el hígado $(50,81,37,30,68,3)$. Los antifólicos actúan inhibiedo esta enzima, de modo que impiden que el A.F. sea reducido a su forma activa, pudiendo producirse como efecto secundario a su uso una A.M. el caso $\mathrm{N}^{\circ} \mathbf{2}$ es un claro ejemplo de este mecanismo, ya que la caída de sus valores hematológicos y los signos morfológicos de megaloblastosis se presentaron en estrecha relación con el uso de amethopterina. Además, se obtuvo una buena respuesta terapéutica con ácido folínico ( $\mathrm{N}-5$ formil-tetrahidrofólico), derivado ya reducido de la vitamina, y que por lo tanto no sufre las consecuencias del bloqueo metabólico $(37,79)$.

Es interesante hacer notar que en la anemia de las enfermedades hepáticas puede jugar un rol la interferencia en la metabolización del A.F., si bien la patogenia de esta anemia es compleja, participando en ella otros factores $(57,83,2)$.

La acción del A.F. adquiere singular importancia en aquellos tejidos en rápida proliferación, como son la mucosa intestinal y la médula hematopoyética. En esta última, la interferencia en la síntesis de nucleoproteínas determina un retardo en el proceso de duplicación de DNA, necesario para la división celular, llegando a producirse las alteraciones megaloblásticas características $(28,60)$. Cuando existe un estímulo para que la médula trabaje a una velocidad mayor que la normal, como ocurre en los procesos hemolíticos crónicos, los requerimientos de A.F. aumentan, pudiendo producirse secundariamente una carencia de esta vitamina. De hecho, se han descrito crisis aplástico-megaloblásticas como complicación de anemias hemolíticas crónicas $(11,15,17,65)$. Nuestro tercer paciente evidencia este mecanismo patogénico, y la extrema gravedad de su cuadro concuerda con la de otros casos publicados (51). Las anemias hemolíticas crónicas en que se ha descrito esta complicación son la Microesferocitosis congénita, la Drepanocitosis y otras hemoglobinopatías $(11,15,51,66)$. En relación a este niño, es de interés destacar que no hemos encontrado referencias bibliográficas de Anemia Hemolítica crónica por déficit de G-6PD complicada de A.M., salvo un paciente descrito anteriormente en Chile (17).

Al analizar el gráfico, hemos destacado los puntos de interferencia en el metabolismo del A.F. Queremos destacar que en la mayoría de los casos la patogenia de la A.M. es múltiple, interviniendo varios de los mecanismos analizados. En el caso No 1, por ejemplo, junto a la falta de aporte dietético puede haber jugado un papel el aumento de los requerimientos determinado por su Infección Urinaria.

En el diagnóstico de la A.M. es fundamental una buena anamnesis, dirigida a descubrir un aporte insuficiente de aquellos alimentos ricos en A.F., antecedentes de diarrea prolongada, infecciones, uso de algunas drogas (anticonvulsivantes, antifólicos), síntomas derivados de hemólisis crónica y de enfermedades hepáticas (30). El examen físico no es específico, demostrando sólo los signos derivados de la anemia y la frecuente coexistencia de desnutrición, diarrea e infecciones $(28,49,70,75,77)$. Los signos hematológicos son característicos, encontrándose anemia, macro-ovalocitosis, hipersegmentación de los neutrófilos, trombopenia y macroplaquetas en sangre periférica. El mielograma muestra hiperplasia eritroide y alteraciones en la maduración de las 3 series precursoras, sobresaliendo la presencia de megaloblastos típicos o intermedios y anomalías de la serie mieloide, fundamentalmente metamielocitos gigantes $(28,51,66$, 70). Ya que estos cambios morfológicos típicos son tardíos en su aparición, para hacer el diagnóstico precoz adquiere gran importancia la hipersegmentación de los neutrófilos en sangre periférica: la presencia de neutrófilos con 6 o más lobulaciones nucleares debe hacer sospechar fuertemente una A.M. Para cuantificar esta alteración se usa la medición del Promedio de Lobulación de los Neutrófilos, que consiste en contar los lóbulos nucleares de 100 neutrófilos sucesivos, dividiendo el total por 100 . Se ha dado como cifra normal $3.17+0.25$, aunque cada laboratorio debe determinar la suya $(28,41$, $38,33,31)$. Consideramos este examen importante de introducir en nuestro medio, por su fácil realización y la imposibilidad hasta ahora de medir el nivel de folato sérico, según la técnica descrita hace más de 10 años (26).

En el diagnóstico diferencial hay que tener en consideración la posibilidad de una Anemia Perniciosa Juvenil Congénita, que como ya expusimos, es extremadamente rara. Este cuadro aparece habitualmente antes de los 2 años (8), coincidiendo con la edad de aparición más frecuente de la A.M. por déficit de A.F., que es entre los 2 y 17 meses, con la mayor incidencia a los 7-8 meses (49). Sirven para diferenciarlos, una cuidadosa historia dietética, la existencia de antecedentes familiares en el caso de una Anemia Per- 
niciosa Juvenil Congénita, la medición de vitamina B 12 y folatos en el suero, y la prueba terapéutica. Esta consiste en usar dosis pequeñas de A.F. $(0.1-0.3 \mathrm{mg} /$ día $)$, que producen respuesta en caso de carencia de A.F., pero son inefectivas si la anemia se debe a déficit de vitamina B $12(28,70,25,30,53$, $56)$.

Dada la alta frecuencia de Anemia Hipocroma Ferropriva en nuestro medio, es muy importante destacar que ella puede enmascarar un déficit de A.F., ya que habitualmente predominan los signos morfológicos de la ferropenia $(11,61,54,33)$. El Promedio de Lobulación de los Neutrófilos ayuda en estos casos a detectar la coexistencia de alteraciones megaloblásticas $(33,31)$. A la inversa, una ferropenia latente puede manifestarse en el período de recuperación de una A.M., retardando la respuesta terapéutica (42), como ocurrió en nuestro primer paciente. Una tercera posibilidad es encontrar un aspecto hematológico mixto (microcitos hipocromos y macrocitos normocromos). Este dimorfismo debe hacer sospechar la existencia de un Síndrome de Malabsorción (80).

El tratamiento de la A.M. por carencia de A.F. consiste en la administración de esta vitamina por vía oral, en dosis de 0.2-1 mg diario, hasta conseguir la normalización de los valores hematológicos, la que se obtiene a las $2-3$ semanas $(28,30,70)$. Entre los 2-5 días de iniciado el tratamiento ya se puede observar, sin embargo, una respuesta reticulocitaria (28). Además, es fundamental aconsejar el aporte de una dieta con alimentos ricos en A.F.

Cuando existen requerimientos aumentados en forma mantenida, como es el caso de las anemias hemolíticas crónicas, debe aportarse A.F. a permanencia (30).

Por último, un caso especial desde el punto de vista terapéutico es el bloqueo metabólico por antifólicos. En estos pacientes se usa un derivado ya reducido del A.F., que existe en la farmacopea como Leucovorina $R$ ( N-5 formil-tetrahidrofólico). Las dosis recomendadas son de $3 \mathrm{mg}$ I.M. cada 12-24 horas $(29,37)$.

Hay que destacar que raramente es preciso usar transfusiones en este tipo de anemia, ya que la respuesta a la terapia específica es muy rápida, incluso en los casos más intensos. Por lo demás, la mortalidad de la A.M., aunque muy baja ( $\pm 2,5 \%)$, puede ser debida en parte a la sobrecarga circulatoria impuesta al emplear este tratamiento (58).

\section{RESUMEN}

Se presentan 3 casos de Anemia Megaloblástica por carencia de Acido Fólico: uno por falla nutricional, otro por uso de antifólicos, y el tercero como complicación de una Anemia Hemolitica Crónica.

Se analiza el metabolismo del A.F., señalando los puntos de interferencia más importantes que pueden conducir a una A.M. Se destaca que la falta de aporte nutricional es el factor etiológico principal en los niños.

Se revisa el cuadro clínico y el diagnóstico y tratamiento de la A.M. por carencia de A.F.

Se considera que es importante tener presente esta afección, dado que su frecuencia no es despreciable en países en desarrollo, especialmente teniendo en cuenta que las alteraciones hematológicas tipicas son tardias. La determinación del Promedio de Lobulación de los Neutrófilos puede constituir en nuestro medio un método fácil de sospecha diagnóstica, ya que no contamos por el momento con otros más especificos, como es la medición de folatos séricos.

\section{SUMMARY}

Three cases of Megaloblastic Anemia due to deficiency of Folic Acid are presented: one caused by nutritional failure, the other one, by the use of antifolic, and the third one, by a complication of a Chronic Hemolytic Anemia.

The metabolism of the Folic Acid is analysed, pointing out the main interference factors leading to a Megaloblastic Anemia. It is stressed that the deficiency of nutritional contribution is the main ethiological factor in children.

The clinical picture, the diagnosis and treatment of Megaloblastic Anemia caused by the lack of Folic Acid are revised.

It is considerel important to keep this disease in mind, since its frecuency is not nebligible in developing countries, mainly taking into account that the typical hacmatological alterations appear rather lately. The determination of the "Lobulation Average of Neutrophiles" may constitute, in our enviroment, an easy method of diagnostic suspicion, since for the moment, we do not have more specific methods, like the measurement of seric folates.

\section{REFERENCIAS}

1.- Baker, H., Frank, O. and Sobotka, H.: Mechanisms of Folic Acid Deficiency in Nontropical Sprue. J.A.M.A., 187: 119, 1964. 
2.- Baker, H., Frank, O., Ziffer, H. y cols.: Effect of Hepatic Disease on Liver B-Complex Vi. tamin Titers. Am. J. Clin. Nutr., 14: 1, 1964.

3.- Baker, H., Frank, O., Feingold, S. y cols.. The fate of orally and parenterally administered folates. Am. J. Clin. Nutr., 17: 88, 1965.

4.- Baker, S.J., Kunar, S. and Swaminathan, S. P.: Excretion of Folic Acid in Bile. Lancet, 1: 685,1965 .

5.- Bernstein, L.H., Gutstein, S., Weiner, S. and Efron, G.: The Absorption and Malabsorption of Folic Acid and its Polyglutamates. Am. J. Med., 48: 570, 1970.

6.- Butterworth, G.E.: Absorption and Malabsorption of Dietary Folate. Am. J, Clin. Nutr., $21: 1121,1968$.

7.- Castle, W.B.: Nutritional Megaloblastic Anemias: Etiological Considerations. Med. Clin. NA., 50: 1245, 1966.

8.- Castle, W.B.: Current Concepts of Pernicious Anemia. Am. J. Med., 48: 541, 1970.

9.- Chanarin, I., Anderson, B.B. and Mollin, D.L.: The Absorption of Folic Acid. Brit. J. Haemat., 4: 156, 1958.

10.- Chanarin, I., Mollin, D.L. and Anderson, B.B.: The Clearance from the Plasma of Folic Acid injected intravenously in normal subjects and patients with Megaloblastic Anemia. Brit. J. Haemat., 4: 435, 1958.

11.- Chanarin, I., Dacie, J.V. and Mollin, D.L.: Folic Acid Deficiency in Haemolitic Anaemia. Brit. J. Haemat., 5 : 245, 1959.

12.- Chanarin, I. and Bennett, M.C.: Absorption of Folic Acid and D-Aylose as Tests of small. Intestinal Function. Brit. Med. J., 1: 985, 1962.

13.-Dahlke, M. and Mertens-Roesler, E.: Malabsorption of Folic Acid due to Diphenylhydantoin. Blood, 30: 341, 1967.

14.- Dawson, D.W.: Partial villous atrophy in nutritional Megaloblastic Anemia corrected by folic acid therapy. J. Clin. Path., 24 : 131, 1971.

15.- Delamore, I.W., Richmond, J. and Davies, S. H.: Megaloblastic Anemia in Congenital Spherocytosis. Brit. Med. J., 1 : 543, 1961.

16.- Dormandy, K.M., Waters, A.H. and Mollin, D.L.: Folic Acid Deficiency in Coeliac Disease. Lancet, 1: 632, 1963.
17.- Ducos, R., Taboada, H., Winter, A. y Flores, H.: Enzimas del Eritrocito. Su estudio en niños normales y en anemias hemolíticas. Rev. Chil. Ped., 39: 381, 1968.

18.- Eichner, E.R., Pierce, I. and Hillman, R.S.: Folate Balance In Dietary-Induced Megaloblastic Anemia New Engl. J. Med., 284: 933, 1971.

19.- Forshaw, J., Moorhouse, E.H. and Harwood, L.: Megaloblastic Anaemia due to dietary deficiency. Lancet, 2: 1004, 1964.

20.- Forshaw, J.: Effect of vitamin B 12 and Folic Acid deficiency on Small intestine absorp. tion. J. Clin. Path., 22, 551, 1969.

21.- Forshaw, J. and Harwood, L.: Diagnostic value of the serum folate assay. J. Clin. Path., 24: $244,1971$.

22.- Ghitis, J.: The labile folate of milk. Am. J. Clin. Nutr., 18: 452, 1966.

23.- Gough, K.R., Read, A.E., Mc Carthy, C.F. and Waters, A.H.: Megaloblastic Anaemia due to Nutritional Deficiency of Folic Acid. Quart. J. Med. 32: 243, 1963.

24.- Gray, O.P. and Butler, E.B.: Megaloblastic Anaemia in Premature Infants. Arch. Dis. Childh., 40:53, 1965.

25.- Hansen, H.A. and Weinfeld, A.: Metabolic Effects and Diagnostic Value of Small Doses of Folic Acid and B12 in Megaloblastic Anaemias. Acta Méd. Scand., 172: 427, 1962.

26.- Herbert, V., Baker, H., Frank, O., Pasher, I., Sobotka, H. and Wasserman, L.R.: The Mesurement of Folic Acid activity in Serum. Blood, 16: 228, 1960.

27.- Herbert, V.: Minimal Daily Adult Folate Requirement. Arch. Int. Med., 110: 649, 1962.

28.- Herbert, V.: Megaloblastic Anaemias - Mechanisms and Management. Disease a Month. August, 1965.

29.- Herbert, V.: Current concepts in Therapy. Megaloblastic Anemia. New Engl. J. Med., 268: 201-368, 1963.

30.- Herbert, V.: Folic Acid. Ann. Rev. Med.: 16: 359, 1965.

31.- Herbert, V.: Biochemical and Hematologic Lesions in Folic Acid Deficiency. Am. J. Clin. Nutr., 20: 562, 1967. 
32.- Herbert, V.: Absorption of vitamin $\mathrm{B} 12$ and Folic Acid. Gastroenterology, 54: 110, 1968.

33.- Herbert, V.: Megaloblastic Anemia as a Problem in world Health. Am. J. Clin. Nutr., 21: $1115,1968$.

34.- Herbert, V.: Nutritional Requirements for vitamin B12 and Folic Acid. Am. J. Clin. Nutr., 21: 743, 1968.

35.- Herbert, V.: Transient (reversible) malabsorption of vitamin B12. Brit. J. Haemat., 17: 213, 1969.

36.- Herbert, V.: Folic Acid Deficiency. Am. J. Clin. Nutr., 23: 841, 1970.

37.- Herbert, V.: Drugs Effective in Megaloblastic Anaemias. Vitamin B12 and Folic Acid. In, The Pharmacological Basis of Therapeutics, 4th ed. (Goodman, L.S. and Gilman, A. eds.). The Macmillan Company, New York, 1970, pp. 1414-1444.

38.- Herbert, V., Chanarin, I. and Woods D.D.: Symposium on Folic Acid Deficiency. Proc. R. Soc. Med., 57: 377, 1964.

39.- Herbert, V. and Zalusky, R.: Interrelations of Vitamin B12 and Folic Acid Mctabolism: Folic Acid clearance studies. J. Clin. Invest., 41: $1263,1962$.

40.- Johns, DG., Hollingsworth, J.W.Cashmore, A. R., Plenderleith, I.H. and Bertino, J.R.: Methotrexate Displacement in Man. J. Clin. Invest., 43: 621, 1964.

41.- Kahn, S.B.: Recent advances in the Nutritional Anemias. Med. Clin. N.A., 54: 631, 1970.

42.- Kende, G., Bracha, R. and Grossowicz, N.: Blood Folic Acid and Vitamin B12 activities in healthy infants and in infants with Nutritional Anemias. Brit. J. Haemat., 9: 328, 1963.

43.- Kho Lien-Keng and Odang, O.: Megaloblastic Anemia in Infancy and Childhood in Djakarta. Am. J. Dis. Child., 97: 209, 1959.

44.- Klipstein, F.A.: Nutritional Folate Deficiency. New Engl. J. Med., 273: 339, 1965.

45.- Klipstein, F.A.: Folate deficiency secondary to disease of the Intestinal Tract. Bull. N.Y. Acad. Med., 42: 638, 1966.

46.- Lazkowsky, Ph.: Congenital Malabsorption of Folate. Am. J. Med., 48: 580, 1970.
47.- Leevy, C.M., Cardi, L., Frank, O., Gellene, R. and Baker, H.: Incidence and Significance of Hypovitaminemia in a Randomly Selected Municipal Hospital Population. Am. J. Clin. Nutr., 17: 259, 1965.

48.- Leikin, S.L.: Pernicious Anemia in Childhood. Pediatrics, 25: 91, 1960.

49.- Luhby, A.L.: Megaloblastic Ancmia in Infancy. III. Clinical Considerations and Analysis. J. Pediat., 54: 617, 1959.

50.- Luhby, A.L. and Cooperman, J.M.: Folic Acid Deficiency in Man and Its Interrelationship with Vitamin B12 Metabolism. Adv. Metab. Dis., $1: 263,1964$.

51.- MacIver, J.E.: Megaloblastic Anaemias. Ped. Clin. N.A., 9: 727, 1962.

52.- Maldonado, N., Fradera, J., Santini, R., Horta, E. and Pérez-Santiago, E.: Hematologic Response to Physiologic Doses of Folic Acid in Tropical Sprue. Am. J. Clin. Nutr., 22: $733,1969$.

53.- Marshall, R.A. and Jandl, J.H.: Responses to "Physiologic" Doses of Folic Acid in the Me. galoblastic Anemias. Arch. Int. Med., 105: 352, 1960.

54.- Matoth, Y., Zmir, R., Bar-Shani, Sh. and Grossowicz, N.: Studics of Folic Acid in Infancy. II. Folic and Folinic Acid Blood Levels in Infants with Diarrhea, Malnutrition, and Infection. Pediatrics, 33: 694, 1964.

55.- Matoth, Y., Pinkas, A. and Sroka, Ch.: Studies on Folic Acid in Infancy. II. Folates in

Breast Fed Infants and their Mothers. Am. J. Clin. Nutr., 16: 356, 1965.

56.- Miller, D.R., Bloom, G.E., Streiff, R.R., LoBuglio, A.F. and Diamond, L.K.: Juvenile "Congenital" Pernicious Anemia. Clnical and Inmunological Studies. New Engl. J. Med., 275: 978, 1966.

57,- Pitcher, C.S. and Williams, R.: Reduced Red Cell Survival in Jaundice and its Relation to Abnormal Glutathione Metabolism. Clin. Sci., 24: $239,1963$.

58.- Polva, I.P. and Kaipinen, WJ.: Mortality in Megaloblastic Anaemia. Lancet, 2: 984, 1970.

59.- Pritchard, J.A., Scott, D.E., Whalley, PJ. and Haling, R.F.: Infants of Mothers with Megaloblastic Anemia due to Folate Deficiency. J.A.M.A., 211 : 1982, 1970. 
60.- Reisner, E.H.: The Nature and Significance of Megaloblastic Blood Formation. Blood, 13: 313, 1958.

61.- Roberts, P.D. and St. John, DJ.B.: Apparent Folate Deficiency in Iron Deficiency Anaemia. Brit. J. Haemat., 20: 165, 1971.

62.- Rosenberg, I.H. and Goolwin, H.A.: The Digestion and Absorption of Dietary Folate. Gastroenterology, 60: 445, 1971.

63.- Santini, $R$. and Horta, E.: Laboratory Criteria for the Diagnosis of Tropical Sprue in Puerto Rico. Am. J. Digest. Dis., 16: 241, 1971.

64.- Saraya, A.K. and Tandon, B.M.: Intestinal structure and function in Megaloblastic Anemia in adults. Am. J. Clin. Nutr., 24: 622, 1971.

65.- Shojania, A.M. and Gross, S.: Hemolytic Anemias and Folic Acid Deficiency in Children. Am. J. Dis. Child., 108: 53, 1964.

66.- Smith, C.H.: Blood Diseases of Infancy and Childhood. The C.V. Mosby Company, U.S.A., 1966, p. 226.

67.- Spurling, C.I., Sacks, M.L. and Jiji, R.M.: Juvenile Pernicious Anemia. New Engl. J. Med., 271: 995, 1964.

68.- Stokstad, E.L.R. and Koch, J.; Folic Acid Metabolism. Phys, Rev., 47: 83, 1967.

69.- Streiff, R.R. and Rosenberg, I.H.: Absorption of Polyglutamic Folic Acid. J. Clin. Invest., 46: 1121, 1967.

70.- Streiff, R.R.: Folic Acid Deficiency Anemia. Semin. Hemat., 7: 23, 1970.

71.- Strelling, M.K., Blackledge, G.D., Goodall, H. B. and Walker, C.H.M.: Megaloblastic Anaemia and Whole-Blood folate levels in premature infants. Lancet, 1: 898, 1966.
72.- Sullivan, L.W.: Differential Diagnosis and Management of the Patient with Megaloblastic Anemia. Am. J. Med., 48: 609, 1970.

73.- Sullivan, L.W., Luhby, A.L. and Streiff, R.R.: Studies of the Daily Requirement for Folic Acid in Infants and the Etiology of Folate Deficiency in Goat's milk Megaloblastic Anemia. Am. J. Clin. Nutr., 18: 311, 1966.

74.- Tasker, P.W.G.: The direct action of Folic Acid, Folinic Acid and Vitamin B12 on Megaloblasts in vivo. Brit. J. Haemat., 2: 205, 1956.

75.- Vildósola, J.: Anemias de patogenia carencial en la infancia. Pediatría, 4: 327, 1961.

76.- Vildósola, J. y Eberhard, R.: Anemia Megaloblástica Infantil. Pediatría, 1: 26, 1958.

77.- Waters, A.H. and Mollin, D.L.: Observations on the Metabolism of Folic Acid in Perni. cious Anemia. Brit. J. Hacmat., 9: 319, 1963.

78.- Waxman, S., Metz, J. and Herbert, V.: Defective DNA synthesis in human Megaloblastic Bone Marrow: effects of homocysteine and methionine. J. Clin. Invest., 48: 284, 1969.

79.- Waxman, S., Corcino, J.J. and Herbert, V.: Drugs, Toxins and Dietary Amino Acids affecting vitamin B12 or Folic Acid Absorption or Utilization. Am. J. Med., 48: 599, 1970.

80.- Whitehead, R., Carter, R.L. and Sharp, A.A.: Anaemia in occult intestinal Malabsorption. J. Clin. Path., 18: 110, 1965.

81.- Whitehead, V.M. and Cooper, B.A.: Absorption of unaltered Folic Acid from the Gastrointestinal tract in Man. Brit, J. Haemat., 13: $679,1967$.

82.- Woodruff, C.: Nutritional Anemias in Early Childhood. Am. J. Clin. Nutr., 22: 504, 1969.

83.- Zieve, L.: Hemolytic Anemia in Liver Disease. Medicine, 45: 497, 1966. 